\title{
DAS THEATER IN ZEITGENÖSSISCHEN ÖFFENTLICHEN INSTITUTIONEN
}

\section{ORIGINAL-ARTIKEL}

JUNIOR, Jorge Luiz Machado ${ }^{1}$

ESTEVES, Alejandra Luisa Magalhães ${ }^{2}$

JUNIOR, Jorge Luiz Machado. ESTEVES, Alejandra Luisa Magalhães. Das Theater in zeitgenössischen öffentlichen Institutionen. Revista Científica Multidisciplinar Núcleo do Conhecimento. Jahrgang 05, Ed. 01, Vol. 01, S. 58-86. Januar 2020. ISSN: 2448-0959, Zugangslink: https://www.nucleodoconhecimento.com.br/businessadministration-de/das-theater-in-zeitgenoessischen

\section{ZUSAMMENFASSUNG}

Die Rechte und die darstellende Kunst sind seit Jahrhunderten zusammen. Die griechischen Tragödien zeigten die Fälle, die vor den Gerichten präsentiert wurden und eine enge Verbindung zwischen den beiden zeigten. Im Laufe der Jahre spiegeln die Theaterstücke oft die Unstimmigkeiten von gerichtsgerichtlichen Entscheidungen oder Gesetzen wider, die ungerecht oder ineffektiv angewandt werden. Die darstellenden Künste werden verwendet, um das Bachelor-Recht zu unterstützen, die Erfahrungen des Berufs durch die Simulation von Gerichten zu erleben, überwacht, wo es einen Bedarf für Inszenierung. Es wird jedoch betont, dass die Absicht ist, den Schüler auf die verschiedenen Situationen vorzubereiten, die der Schüler durch die Ausübung seiner Aktivitäten durchlaufen kann. In diesem Zusammenhang werden

${ }^{1}$ Bachelor-Abschluss in Public Administration 7. Periode und Technologe in Public Management.

2 Doktortitel in Graduiertenprogramm in Soziologie und Anthropologie. Master in Sozialgeschichte. Spezialisierung in der Geschichte Afrikas und Schwarz in Brasilien. Abschluss in Geschichte. 
öffentliche Institutionen und ihre Besonderheiten beachtet. Diese zeichnen angesichts der derzeit auftretenden Probleme wie Korruption und Ineffizienz der Unternehmensführung ein abgenutztes Image auf, die bei der Suche nach möglichen Lösungen für die Langsamkeit bei der Erbringung von Dienstleistungen stärker angegangen werden. Schließlich wird darauf hingewiesen, dass es die Möglichkeit gibt, Theater und öffentliche Einrichtungen in Beziehung zu setzen, um die Rolle seiner Akteure bei der Verwaltung der erbrachten Dienstleistungen aufzuklären.

Stichworte: Theater, öffentliche Institution, Langsamkeit.

\section{EINFÜHRUNG}

Theater und Recht gehen in seinen verschiedenen Tätigkeitsbereichen Hand in Hand. In einigen Fällen können die darstellenden Künste bei der Einfallsreichtum der Studenten vor dem Richter helfen, ihre Räumlichkeiten zu offenbaren.

Der Begriff Theater hat eine griechische théatron etymologis che Grundlage, die Kunst konfiguriert, wo ein Schauspieler oder eine Gruppe von Schauspielern eine Geschichte für ein Publikum an einem bestimmten Ort darstellt.

Das Theater stammt aus dem antiken Griechenland im 4. Jahrhundert v. Chr. als Ergebnis von Festen, um den Gott der Freude und Fruchtbarkeit zu preisen, Dionysos. Die darstellenden Künste wurden nur von Männern mit großen Masken vertreten, weil Frauen nicht als Teil der Gesellschaft betrachtet wurden. Auch in Griechenland kamen zwei Stränge der Theaterkomödie und Tragödie.

Eine kurze Parallele zu öffentlichen Organisationen und darstellenden Künsten findet sich in dem Film "The Time Breakdown", in dem Regisseur Santiago Dellape die Wahrheit der brasilianischen öffentlichen Institutionen mit wenigen Mitarbeitern oder mit Nebendarstellern porträtiert. , bekannt als "Aponen". Obwohl es kein Theaterstück ist, lohnt es sich, die Bedeutung des Werkes durch seinen aktuellen und kritischen Charakter zu betonen. 
Der Spielfilm spielt in den 1980er Jahren in Brasilia. Das für die Erfassung von Erfindungen und Patenten zuständige öffentliche Amt zeigt eine Langsamkeit bei der Ausführung seiner Dienste, wenn eine Maschine eintrifft, mit einem Punktuhr-Aspekt, der eine Zeitreise auf der Suche nach einer besseren Nutzung ermöglicht. öffentlichen Dienst, der als fahrlässig und desinteressiert angesehen wird. Es ist jedoch darauf hinzuweisen, dass die bürokratischen Hindernisse, die dem System innewohnen, die Kontinuität des Spektakels behindern. Die tragischen alten Stücke zeigten Themen im Zusammenhang mit Gerechtigkeit und Normen.

Von den Revolutionen, die in Europa im 17. und 19. Jahrhundert stattfanden, und der Erosion der Bourgeoisie, haben die Stücke Einflüsse aus dem historischen Moment gelitten und präsentieren einen individuelleren Charakter, der ihre soziale Natur verliert.

Die Einflüsse der romantischen Bewegung brachten das Motto der Französischen Revolution in die Stücke: Brüderlichkeit, Gleichheit und Freiheit. Im 20. Jahrhundert wird das Theater zu einem Werkzeug der Gesellschaft für Debatten und Kritik, ohne große Aufmerksamkeit die Allegorien der Szenarien und Kostüme, mit der Absicht, die soziale Realität zu klären.

Laut Teixeira (2018) gibt es Beweise dafür, dass Theater im antiken Griechenland in der Ähnlichkeit der Gerichte entstanden sind und auch als Vertreter der Justizorganisation eingesetzt wurden, was es den Menschen ermöglichte, ihre Verbrecher durch ihre Inszenierungen zu beurteilen. Es verdeutlicht durch die Worte von Jeniffer Wise, Professor für Theatergeschichte an der Universität von Victoria, die beide reflektieren "Demokratie in Aktion" und bieten eine Bewertung der rechtlichen Normen mit den konkreten Erfahrungen der Gesellschaft.

Ziel dieses Artikels ist es daher, eine Reflexion über den Leser zu diesem Thema vorzuschlagen. Die darstellenden Künste, die von ihrer Entstehung bis zur Gegenwart als Instrument für Gesellschaftskritik und politisches Bewusstsein genutzt wurden, auch mit dem Ziel, in die Rechte für die Ausarbeitung und Änderung von Gesetzen für 
die Gesellschaft und in die Entwicklung von Servern einzugreifen die sich verändernden Konstaaten zeitgenössischer öffentlicher Institutionen repräsentieren.

Derzeit ist Kunst eine universelle Sprache, die die markanten Aspekte der Gesellschaft weiterhin präsentiert. Dolmetschen fördert das Wissen über innovative Dynamik und unterscheidet sich vom Altag des Dolmetschers. Es ist zwingend notwendig, die Artikulation zwischen Theater und öffentlichen Einrichtungen hervorzuheben, um Konzepte und Funktionen zu identifizieren und vertraut zu machen, indem man sie leichtfertig einsetzt, kritisiert aber die von innen dargestellte Unwirksamkeit.

\section{2. ÖFFENTLICHE INSTITUTIONEN}

Die Institutionen wurden konzipiert, im alten römischen Gesetz mit dem Plan, den Willen des Gründers zu erfüllen, um seine Ziele zu erreichen. Im unteren Römischen Reich entstanden durch christliche Ideologie, die Nächstenliebe predigte, die ursprünglichen Grundlagen. Beim Verständnis der individualistischen Theorie in Bobbios Vision (1992) besitzen Männer die Rechte und Empfänger dieser Rechte, in den Stiftungen, sind diejenigen, für die sie entstanden sind.

Im Mittelalter handelten die Stiftungen unter der Unterstützung der Kirche und hatten einen öffentlichen Charakter, der mit dem Ursprung der heutigen Nationen und der inhärenten Relevanz für das Zivilrecht reduziert wurde.

Das Dekret Nr. 200/67 stellte fest, dass die Stiftungen öffentlichen Gesellschaften ähnlich seien und indirekte öffentliche Verwaltungen einbauten. Andere Dekrete stellten später die Positionierung öffentlicher Einrichtungen als Teil der indirekten Verwaltung in Frage.

Dekret-Gesetz Nr. 2,299/86, fügte Absatz 2, er umrahmte die Institutionen in der Liste der indirekten Verwaltung zu den generierten, in der Absicht, sie zu Werkzeugen und Richtlinien für die Inspektion, Kontrolle und Finanzmanagement zu bestechen, zusätzlich zu dem Karriereplan durch Gesetz Nr. 5,645/70.com Aufkommen von Änderungen, durch das Gesetz, die Stiftungen präsentierten einen überwiegend 
öffentlich-rechtlichen Charakter, weil sie nicht mehrere zivilistische Gesetze zu erlassen. In dieser Regel wurden die Einheiten dem Bürgerlichen Gesetzbuch nur in Bezug auf die Form der Verfassung untergegnet.

Die verfassungsmäßige Literalität unterschied öffentliche und private Stiftungen. Mit dem Aufkommen der Verfassungsänderung Nr. 19/98 verwendete sie nicht mehr den Ausdruck öffentliche Stiftung die Änderungen klarstellen, dass die Verfassung nicht unterscheidet, daher die Normen der Magna Carta umfassen alle Arten von Stiftung.

Kunst. 5o Für die Zwecke dieses Gesetzes gilt es als:

IV - Öffentliche Stiftung - die Einrichtung mit einer Rechtspersönlichkeit des privaten, gemeinnützigen Rechts, die aufgrund der gesetzlichen Ermächtigung geschaffen wurde, für die Entwicklung von Tätigkeiten, die keine Ausführung durch Organe oder Einrichtungen des öffentlichen Rechts erfordern, mit Verwaltungsautonomie, Eigenkapital, das von den jeweiligen Leitungsorganen verwaltet wird, und Operationen, die aus Mitteln der Union und anderer Quellen finanziert werden (BRASIL, 1988, S. 13)

Körperschaften des öffentlichen Rechts stammen aus dem Gesetz, während die im Privatrecht geschaffenen Personen durch die rechtliche Genehmigung als Zivilrechtsvoraussetzungen entstehen. Beides, den Erwerb von Rechten und Pflichten in ihrem eigenen Namen. Nach Angaben des Obersten Gerichtshofs in der direkten Aktion der Verfassungswidrigkeit 191/RS, in der die Berichterstatterin Ministerin Carmen Lucia war. Die Formen, in denen die Stiftungen geschaffen wurden, weichen aufgrund des Eigentums und der Art der erbrachten Dienstleistungen von der Öffentlichkeit ab, die privaten Stiftungen (ADMIN, 2008).

Was die Verwaltungsvorschriften betrifft, so halten sich die öffentlichen Stellen der indirekten Verwaltung an die Ihrer Persönlichkeit angemessenen Regeln der öffentlichen Verwaltung, die öffentlich oder privat sind. (CARVALHO FILHO, 2009). 
Auf diese Weise erklärt Kunst. 37 der Magna Carta:

Kunst. 37. Die direkte und indirekte öffentliche Verwaltung einer der Mächte der Union, der Länder, des Bundes und der Kommunen wird die Grundsätze der Legalität, Unpersönlichkeit, Moral, Publizität und Effizienz sowie folgendes einhalten:

XIX - nur durch spezifisches Recht kann die Institution der öffentlichen Gesellschaft, gemischte Wirtschaft und Stiftung geschaffen werden, mit dem ergänzenden Gesetz, in diesem Fall, die Bereiche ihrer Tätigkeit zu definieren. (BRASIL, 1988, p. 36 -37).

Daher können sich öffentliche Organisationen aus den Behörden zusammensetzen, deren Kapital ganz oder teilweise öffentlich ist, von der öffentlichen Verwaltung beaufsichtigt wird, mit der Möglichkeit der Selbstverwaltung im Rahmen der Norm.

Das Vermögen von Öffentlich-Rechtlichen wird als öffentliches Gut bezeichnet, das durch alle Privilegien geschützt ist, wie z. B. die Undurchsetzbarkeit von Vermögenswerten. Das Vermögen der privatrechtlichen Institutionen passt nicht als öffentliche Ware.

Die Veränderungen im Umfang der öffentlichen Verwaltung in Brasilien können bei der Suche nach der Wiederbelebung staatlicher Maßnahmen identifiziert werden, heißt es, Verbesserungen in der Leistungsfähigkeit der erbrachten Dienstleistungen sowie die Entstehung neuer Leitlinien in der Beziehung mit dem (MOTTA, 2007). Die Umsetzung dieser Änderungen leidet jedoch unter der Aufrechterhaltung klassischer Aspekte der öffentlichen Verwaltung. Als ein Stück, das lange mit großer Akzeptanz beim Publikum zu sehen ist, wo Schauspieler das Drehbuch ändern wollen, improvisieren, aber der Autor es nicht zulässt.

Früher war die von den Beamten dieser Stiftungen gewählte Rechtsordnung die gleiche, wie es in der Kunst heißt. 39 der CF, die die einheitliche Rechtsordnung einführte. Mit der Einführung der EG-Nr. 19/98 wurde dieses System abgeschafft, und 
das Personalregime wurde das System, das von der durch die Gesetzgebung geschaffenen föderativen Person verwendet wurde. Beide Einheiten haben objektive Verantwortung, weil Kunst. 37, Nr. 6 der Verfassung erläutert (BRAZIL, 1988).

Organisationskultur ist ein relevantes Element für das Verständnis der Leistung von Organisationen, insbesondere nach Interventionen. Dieser Vorschlag zielt darauf ab, Versäumnisse und Maßnahmen des Staates neu zu definieren und signifikante Änderungen in der Verwaltung öffentlicher Einrichtungen zu erreichen, so Silva und Fadul (2010).

Die Krise, mit der der Staat konfrontiert ist, und die Glaubwürdigkeit derjenigen, die verschiedene öffentliche Organisationen leiten, erforderten einen internen Wiederaufbau, um die Eigentumsverhältnisse zu gewährleisten, und halfen nicht, die wirtschaftliche Koordinierung auf dem Markt zu unterstützen und Ungleichheiten zu verringern. sozial. Dieser Plan zielt darauf $a b$, managern durchsetzungsfähige Entscheidungen zu ermöglichen und die Dienstleistungen, die ihm direkt oder indirekt untergeordnet sind, effektiver zu gestalten.

Die Globalisierung hat es relevant gemacht, Ihre Aufgaben neu zu definieren. Zuvor hatten die Staaten die globale Einbeziehung von Märkten und Produktionsweisen als eines ihrer grundlegenden Ziele, ihre Volkswirtschaften des internationalen Wettbewerbs zu schützen. Nach dem Globalisierungsprozess konnte der Staat diese Haltung nicht beibehalten, sondern ein starkes internes Wirtschaftssystem einführen, das inn international wettbewerbsfähig macht.

Hinzu kommt der große soziale Rückschlag, der von der neoliberalen Regierung verursacht wurde, da wir es mit dem postdemokratischen Staat zu sehen haben, in dem alles zur Ware wird und politische Entscheidungen von der Richtung großer transnationaler Konzerne getroffen werden, Märkte, unter anderem. So üben öffentliche Einrichtungen ihre Funktionen nicht umfassend aus, wenn sie nicht im Rahmen der Sehnsehnsehnsehnsehnungen des Neoliberalismus handeln, was ihre 
Verachtung für die Prozesse der Volkslegitimation sowie ihre tiefe Auseinandersetzung mit dem Finanzkapital zeigt.

Der Unterschied zwischen einem neoliberalen Reformplan und einem demokratischen Sozialplan ist das Ziel. Im ersten wird versucht, sich aus der Wirtschaft zurückzuziehen, während in der zweiten Absicht die Regierung des Staates zu erhöhen ist.

Das Verständnis der Art der Krise und der unverzichtbaren Notwendigkeit, Reformen im Staat umzusetzen, geschah auf unvorhergesehene und kontroverse Weise. Das Land litt von 1979 bis 1994 unter einer Phase der Kristallisation des Pro-KopfEinkommens und einer tiefen Inflation.

Was diese Krise in der Wirtschaft verursachte, war die Staatskrise, die trotz der Bemühungen, sie zu beenden, bis heute andauert. Die 1979 eingeleitete Depression wurde durch die zweite Ölkrise ausgelöst, die durch den Mangel an staatlicher Fähigkeit zur Strukturierung des Wirtschaftssystems zur Integration des Marktes identifiziert wird. Diese Krise ist definiert als eine Neusektion mehrerer Fronten: fiskalisch, politisch, die Methode der staatlichen Einmischung, der staatlichen Verwaltung. Mit dem Aufkommen des Königlichen Plans im Jahr 1994 stabilisierten sich die Preise zugunsten der Erholung des Wirtschaftswachstums.

Die politische Instabilität stellte laut Pereira (1996) drei Perioden dar: eine Krise des Militärsystems; und eine moralische Krise, die zu Collors Amtsenthebung führte; die Instabilität der Interventionsmethode, die durch die globale wirtschaftliche Globalisierung erweitert wurde, wurde durch die Schwächung der protektionistischen Art des Importaustauschs qualifiziert, was sich in Ermangelung der Wettbewerbsfähigkeit eines großen Teils der Unternehmen zeigte. Brasilianische Frauen; er erschien in dem Versäumnis, Brasilien näher an europäische sozialdemokratische Modelle zu bringen. 
Die Verfassung von 1988 brachte die Verwaltung auf die entgegengesetzte Ebene: eine intensive bürokratische Starrheit. Die Ergebnisse sind hohe Kosten und eine geringere Qualität der brasilianischen Verwaltung.

Diese Reform zielte darauf $\mathrm{ab}$, bessere Bedingungen für die Anwendung von Gesetzen und öffentlichen Politiken zu schaffen und die ausschließlichen Aktivitäten des Staates durch den Wechsel der Gemeinden in "autonome Agenturen" zu verbessern und wettbewerbsfähige soziale Dienstleistungen in sozialen Organisationen.

Im Staat gab es keine neuen öffentlichen Ausschreibungen, da Berechnungen vorgelegt wurden, bei denen es mehr inaktive Server als Vermögenswerte gab (MOTTA, 2007). Unterdessen war der Staat mit reduzierten Humanressourcen der Haushaltskrise ausgeliefert. Nach der (BRASIL, PDRA, 1995, S.26) "verhindert die Starrheit der Stabilität, die den Beamten gewährleistet ist, die Angemessenheit des Personals für die tatsächlichen Bedürfnisse des Dienstes und erschwert die Abholung der Arbeit".

Das monetäre Verfahren der Union ist durch Asymmetrie definiert, weil es in Brasilien kein einheitliches Vergütungssystem gibt. Die Reformplanung wurde von der britischen Regierung inspiriert und zielte darauf ab, einen neuen Standard der öffentlichen Verwaltung zu finden, der auf der Forderung nach schnellerer Und-Orientierung mit einem Fokus auf Ergebnisse, die öffentliche und soziale Kontrolle basiert.

Die Steuerregelung wird durch die Befreiung von Überschussservern, die Abgrenzung der Gehaltsobergrenze der Arbeitnehmer und die Änderung des Pensionierungsprozesses mit der Erhöhung der Dienstzeit und das Mindestalter für den Ruhestand mit einer Mindestdauer von Leistung und Wert proportional zum Beitrag.

Die Modernisierung der öffentlichen Verwaltung wird als Folge des Reformplans vorgestellt, der darauf abzielen wird, das strategische Zentrum des Staates zu stärken und die öffentliche Verwaltung durch die Einführung anderer Zentren, die durch 
Managementverträge kontrolliert werden, zu disfokussieren. Mit diesem Plan soll die zentrale Verwaltungskompetenz neu belebt und Agenturen und sozialen Organisationen Autonomie geboten werden. Um mit der Einführung der Reform einen Triumph zu erreichen, ist das Engagement aller Akteure und Regisseure, die in allen Bereichen innerhalb ihrer spezifischen Vorrechte tätig sind, unerlässlich.

Es lohnt sich, darüber nachzudenken, wie solche Ideen akzeptiert und in der Routine öffentlicher Organisationen eingesetzt werden. Wie Motta (2007) erklärt, ist die öffentliche Verwaltung mit der berühmten Kultur korreliert, und solche Reformen dürfen diese Verbindung nicht sofort unterbrechen. In diesem Zusammenhang fügt er hinzu, dass Patrimonialismus und Personalismus fortbestehen und die Konventionen der Erhaltung von Machtkoalitionen und der Wahrung von Zielen der präferenziellen Kollektivitäten leiten.

Durch die gemeinsame Erfahrung der Teams von Organisationen sind Schlussfolgerungen zu den Routineproblemen des Unternehmens, weil sie gemeinsame Muster und Meinungen sind, die den operativen Modus bestimmen. Diese gemeinsamen Muster und Meinungen bilden die Organisationskultur (MOTTA, VASCONCELOS, 2006).

Die Voraussetzung, ein positives Konzept zu entwickeln, ist zur Hauptgrundlage von Verwaltungen geworden, die qualität wollen. Anstatt Investitionen in die Umstrukturierung des öffentlichen Staatsapparats oder in Projekte und Dienstleistungen zu tätigen, wird eine große Menge an Ressourcen ausgezahlt, um die Realität von Korruption, Verschrottung, Vetternwirtschaftszulage zu verschleiern. Ineffektivität unterstützt sie und deckt sie von Anfang an von brasilianischen öffentlichen Einrichtungen an ab. (SCHAUN, 1986).

Die intrinsischen Aktivitäten des Staates, so heißt es, das Vorrecht, Gesetze zu erlassen und zu besteuern, werden durchgeführt und umfassen die Polizei, die Streitkräfte, die Aufsichts- und Regulierungsstellen sowie die mit der Zuweisung von Ressourcen betrauten Stellen. 
Nicht-peculiar Dienstleistungen, die der Staat ausübt und/oder fördert, weil sie sie für äußerst relevant für die Menschenrechte halten oder externe Volkswirtschaften abdecken.

Schließlich sollte man in jedem Sektor analysieren, welche Art von Natur ist und welche Art von öffentlicher Verwaltung am besten geeignet ist.

Die Komplexität der Institutionen und der Reform ist ein Thema, das einer Analyse verschiedener Punkte in der Geschichte des Landes bedarf. Es ist keine einfache administrative Frage der Verschlechterung öffentlicher Einrichtungen, und um diesen Prozess zu verstehen, sollten drei Gründe analysiert werden, die: die brasilianische Kultur; Korruption, die der Regierung innewohnt, und die Unfähigkeit des Regierungsapparats.

\subsection{ABBAUFAKTOREN ÖFFENTLICHER EINRICHTUNGEN}

a. Brasilianische Kultur

Nelson Werneck Sodré (1996) verrät in seinem Werk "Synthese der Geschichte der brasilianischen Kultur", dass die ersten Kulte des Landes Jesuiten waren. Im Religionsunterricht wurden die Lehren mit dem wesentlichen Ziel der Katechese übertragen. Nach vielen Jahren nach diesem System wird die Pombaline-Reform durch die Notwendigkeit des Verbots der Jesuiten erzeugt, was nicht zur Ausarbeitung einer neuen pädagogischen Methode führt und dem Rückgang des Unterrichts in Brasilien zugute kommt. Priester und Kaplan waren die einzigen, die Wissen hatten, aber ohne pädagogische Grundlage. Der Bildungsprozess war für die wenigen, und die Schlussfolgerung fand in der Regel in europäischen Ländern statt.

Kultur, so Sodré (1996), zielte darauf ab, das Diplom zu erlangen, nicht wissend, und überlegte, dass seine Studenten Prediger, Kulte und Genies sein würden und dass sie daher nach freien Berufen und öffentlichen Berufen strebten. Die Kultur der Indianer und Schwarzen war eng und diese durften nicht lernen, eine Tatsache, die ihre Fortschritte verhinderte. Diese Divergenz zwischen fortgeschrittener politischer 
Abstraktion und der Verzögerung der Umwelt zeigte den Punkt der kulturellen Desorientierung, die Völkern mit kolonialen Vorfahren innewohnt.

Die Folge dieser Tatsachen war eine mittelmäßige Grundschule seitens des Staates und ein Gymnasium, das privaten Organisationen zugeteilt wurde, die den bescheidensten Klassen der Bevölkerung finanziellen und immateriellen Charakter verliehen. In der Mitte des 21. Jahrhunderts hat Brasilien nach IBGE-Daten eine Analphabetenrate von $7 \%$ bei Menschen ab 15 Jahren.( Ein Index, der noch weit von dem der Industrieländer entfernt ist und einige in der Entwicklung, wie Korea, das in 45 Jahren den Analphabetismus praktisch ausgerottet und $82 \%$ der jungen Menschen an die Universität gebracht hat. (MAZILLI, 2011).

Nelson Sodré (1996) präsentiert eine relevante Analyse der Medieneinmischung in den Bildungsprozess der Bevölkerung:

Unsere Leut[...]e werden immer noch durch das niedrige Niveau angeklagt; kommerzielle und ideologische Entdecker der Massenmedien wären bloße Opfer dieses unverbesserlichen Volksgeschmacks; Letztendlich liefern sie nur das, was innen durch die Anforderungen dieses schlechten Geschmacks aufgezwungen wird. Dieser Betrug stößt an die Grenzen des Spotts, wenn bekannt ist, dass die Wahrheit eine ganz andere ist: Die Öffentlichkeit akzeptiert und sucht das Beste. (SODRÉ, 1996, p.79).

Angesichts dieses Panoramas der Ignoranz scheint es unwahrscheinlich, dass die brasilianische Bevölkerung die Überlegungen des Systems bekämpfen wird, jedoch öffentliche Politiken, die darauf abzielen, zum Eintritt der weniger begünstigten Klassen in die Hochschulbildung und in den Lehrplanänderungen beizutragen. in der Grund- und Oberschule zielen sie darauf ab, das Gleichgewicht des Wissens für alle auszugleichen. 
Während in anderen Zeiten die Presse als Bewusstsein der Bevölkerung agierte, fragen und rebellieren die Menschen jetzt gegen die Presse, vielleicht durch das Aufkommen des Internets, aber vor allem durch das Erwachen des Wissens.

b. Korruption

Korruption ist ein häufiges Thema in allen Medien und wird als einer der Faktoren dargestellt, die zu einer Verschlechterung des Rufs öffentlicher Einrichtungen führen. In ähnlicher Weise haben sich Studien zu diesem Thema ausgeweitet und betrachten dies größtenteils als moralische Frage. Bezerra (1995) konzipiert in seinem Werk "Korruption: eine Studie über öffentliche Macht und persönliche Beziehungen in Brasilien" Korruption als "Phänomen struktureller Natur, das in unserer sozialen Bildung, in unseren Gewohnheiten und Bräuchen verwurzelt ist".

Die Denunziationen der Korruption waren häufiger und werden von den Medien als ethische Krise dargestellt. Um das Problem zu lösen, müssen strengere und ethischere moralische Standards festgelegt werden (BEZERRA, 1995).

Der Autor verbindet Korruption und persönliche Beziehungen und Netzwerke. Ihm zufolge sind Bande der Freundschaft, Verwandtschaft oder Mäzenatentum Ansaton, um Korruptionshandlungen zu rechtfertigen.

Stukart (1988) betonte diesen Zusammenhang zwischen korrupten Handlungen angesichts der sozialen Beziehungen: "Zweifellos sind moralisch apokryphen Privilegien und Treuhandschaften auch ein Mittel der Bestechung." Bezerra (1995) ergänzt:

Es sind nicht endlich außergewöhnliche Tatsachen, sondern Routinepraktiken. Es sei auch darauf hingewiesen, dass eine rasche Beobachtung der Korruptionsvorwürfe in den letzten Jahren es ermöglicht, festzustellen, dass das so bezeichnete Verhalten nicht ausschließlich einem Zeitraum unterliegt, sondern verschiedene 
politische Gruppen und Regierungen reproduziert und durchquert hat (BEZERRA, 1995, S. 186).

Der Autor stellt klar, dass Korruption Teil der Kultur der brasilianischen Bevölkerung ist und dass selbst diejenigen, die gegen diese Praktiken sind, wenn sie Status erreichen, persönliche Beziehungen bevorzugen.

[...] Wir lesen fast täglich in Zeitungen und Zeitschriften Fälle von Korruption, aber selten lesen über die Entschädigung des Schadens und / oder Bestrafung der korrupten, so dass der Eindruck, dass wir ein Syndrom der Straflosigkeit leiden, was einige zu dem Schluss führen, dass wirklich "Verbrechen zahlt sich aus. (STUKART, 1988, p.52).

Diese Taten werden allmählich stärker der Gesellschaft ausgesetzt und tragen zum Image der Prekarität des gesamten Regierungssystems bei. Um diesen Rahmen zu ändern, ist die Haltung der Gesellschaft in der Konfrontation gegen die Korruption unerlässlich. Die ständigen sozialen Anstrengungen und die Verurteilung von Korrupten können große Veränderungen zur Verringerung oder sogar Ausrottung der Korruption im Land mit sich bringen (STUKART, 1988).

Der Autor fügt hinzu: Der Ethikkodex, der nach dem Überlebensbedürfnis einer Gruppe geschaffen wurde, braucht Sanktionen und Strafen, damit er nicht nur ein Haufen bloßer leerer Worte ist. Nachlässigkeit bei ihrer Anwendung wird ihr allmähliches Verschwinden bestimmen. (STUKART, 1988).

Jüngsten Daten des Institute of Transparency International zufolge belegte Brasilien mit 17 Stellen im Vergleich zur vorherigen Studie den 96. Platz in den am wenigsten korrupten Ländern der Welt. Die meisten Gebiete, die als korrupter gelten, leben in einem diktatorischen politischen System oder präsentieren religiöse Konflikte und schlechte Lebensbedingungen der Bevölkerung. (EXAME, 2018) 
c. Betriebsinsinder

Es ist wichtig, den Staat zu organisieren, die bürokratischen Systeme der Vergangenheit aufzugeben, damit er Managementmethoden etablieren kann, die im Verhalten der öffentlichen Arbeit zwingende Konzepte von Qualität, Produktivität und Verantwortung etablieren nach Pereira und Spink (1998, S. 23-24).

Die Bürger verlangen viel mehr vom Staat, als der Staat bieten kann. In diesem Fall wird die Funktion einer effizienten öffentlichen Verwaltung zu einem strategischen Wert, der die Kluft verringert, die die soziale Nachfrage und die Befriedigung dieser Nachfrage trennt. Das Ziel ist es, einen Staat zu schaffen, der auf die Bedürfnisse seiner Bürger eingeht; demokratischer Staat, in dem es Politikern möglich ist, die Leistung von Bürokraten zu überwachen, und deren Leistung gesetzlich vorgeschrieben ist, für sie Rechenschaft abzunehmen, und in dem die Wähler die Leistung von Politikern überwachen können und sie auch gesetzlich verpflichtet sind, Konto.

Die oben genannten Autoren erklären, dass die bürokratische Krise der brasilianischen öffentlichen Verwaltung vom Militärregime herrührte, angesichts des Patrimonialismus, der die Konsolidierung einer professionellen Bürokratie verhinderte, die Manager über staatliche Unternehmen rekrutierte. Die Magna Carta von 1988 verunglimpfte die neuen Richtlinien der öffentlichen Verwaltung.

Die Wähler und im weiteren Sinne die brasilianische Gesellschaft haben in diesem Moment einen unglaublichen Mangel an Fähigkeit offenbart, das Neue zu sehen. Sie erkannten nur, dass die klassische bürokratische Verwaltung, die in den 1930er Jahren im Land eingeführt worden war, nicht vollständig etabliert war. Sie sahen, dass der Staat dezentrale Strategien - die Kommunen und öffentlichen Stiftungen - angenommen hatte, die nicht zum klassischen bürokratisch-professionellen Modell passten. Sie bemerkten, dass diese Dezentralisierung Raum für 
Klientelismus eröffnet hatte, vor allem in den Staaten und Gemeinden Klientelismus, der nach der Redemokratisierung zugenommen hatte. Sie erkannten jedoch nicht, dass die dezentraleren und flexibleren Formen der Verwaltung, die das Dekret Nr. 200/1967 verankert hatte, eine Antwort auf die Notwendigkeit für den Staat waren, Unternehmen und soziale Dienstleistungen effizient zu verwalten. Und sie beschlossen, die bürokratische Revolution zu vollenden, bevor sie über die Prinzipien der modernen öffentlichen Verwaltung nachdachten. Indem sie so handelten, folgten sie offenbar einer linearen Logik, die mit der Vorstellung vereinbar war, dass es zunächst notwendig wäre, die mechanische Revolution zu vollenden, um nur dann an der elektronischen Revolution teilzunehmen. (PEREIRA e SPINK, 1999, p.246).

Viele Politikwissenschaftler definieren, wie wichtig es ist, durch die öffentliche Maschinerie des Staates die Wirksamkeit des Privatsektors und die Verwendung von Konzepten wie Qualität und Agilität bei der Erbringung ihrer Dienstleistungen zu übernehmen. In diesem Zusammenhang reichen die Maßnahmen von der Privatisierung öffentlicher Organisationen bis zur Einführung einer hybriden Verwaltungsmethode, die von Ferlie et al. (1999) unterstützt wird.

Nach Ferlie et al(1999) ist die vorgeschlagene hybride Methode weder zuverlässig noch logisch, sondern könnte unter Beachtung von zwei Elementen funktionieren: der Steuererhebung und dem privaten Markt.

Eine Analyse der Interamerikanischen Entwicklungsbank IDB zeigt, wie viel Zeit, Geld und Produktivität angesichts der Bürokratie in lateinamerikanischen Ländern verschwendet werden. Der Mangel an Investitionen untergräbt die höhere Geschwindigkeit im öffentlichen Dienst. Online-Verfahren nehmen 74 \% weniger als persönliche Verfahren, um die Kosten abzuschließen und zu senken, auch Dokumente zu digitalisieren und die Häufigkeit von Korruption zu verringern. "Der Durchschnitt für ein einzelnes Verfahren beträgt 5,4 Stunden, aber einige Nationen haben Anrufe, die mehr als 11 Stunden dauern." (IDB, 2018, S.4) 
Bürokratische Verfahren sind in der Region schwierig: Sie sind langsam, korruptionsanfällig und schließen am Ende Menschen mit weniger Ressourcen aus. Viele von ihnen sind immer noch von Angesicht zu Angesicht und beinhalten Papierdokumente. Die Bürger verschwenden Zeit damit, von Dertheke zu gegensteuern und zahlen in vielen Fällen Bestechungsgelder an Server. Unternehmen verlieren produktive Stunden und damit einen Teil ihrer Wettbewerbsfähigkeit. Der Staat geht in komplexen und manuellen Verfahren verloren und kann öffentliche Maßnahmen nicht mit den beabsichtigten Begünstigten verbinden (IDB, 2018, S.2 )

Die Kosten für die persönliche Pflege kosten bis zu 40-mal mehr als ein computergestützter Dienst an die Regierung. Brasilien ist eines der Länder, in denen innovativere Technologien im öffentlichen Sektor eingesetzt werden. Leider reicht es immer noch nicht aus, beispielsweise die Europäische Union zu erreichen, die $81 \%$ der COMPUTERdienste hat. Was die Qualität betrifft, so liegt sie in den lateinamerikanischen Ländern weit unter dem Erwarteten. Nur die Hälfte der Verfahren wird in einer einzigen Interaktion mit der öffentlichen Stelle gelöst, und $25 \%$ von ihnen erfordern drei Oder mehr Interaktionen, was beim Zugang zu grundlegenden Dienstleistungen wie Bildung, Gesundheit, Steuerzahlung und Geburtsurkunde (FERRARI, 2018)

In diesem Zusammenhang "benötigt Brasilien durchschnittlich 5,4 Stunden, um bürokratische Verfahren zu lösen" (IDB, 2018. p.4). Antagonistische Ideologie, dass der Staat als privates Unternehmen bei der Erbringung von Dienstleistungen agieren muss, sind Osborne und Gaebler. Die Autoren weisen auf die Unterschiede zwischen öffentlichen und privaten Institutionen hervor, die mit der Anwendung einer einzigen Methode der Verwaltung im Einklang stehen.

Die Regierung ist demokratisch und offen; ihre Bewegungen sind also langsamer als die von Unternehmen, deren Verwalter hinter verschlossenen Türen schnelle Entscheidungen treffen können. Die 
grundlegende Aufgabe der Regierung ist es, "Gutes zu tun", es ist nicht "Geld verdienen". [...]. Diese Unterschiede führen zu einer Schlussfolgerung: Man kann nicht regieren, wer ein Unternehmen führt, obwohl es sicherlich viele Ähnlichkeiten zwischen den beiden Aktivitäten gibt. (OSBORNE e GAEBLER, 1994, p.22).

Diese Autoren erklären, dass jede Institution, ob öffentlich oder privat, sich verpflichten oder bürokratisch sein kann. In Brasilien sind öffentliche Einrichtungen extrem bürokratisch, was Verwaltungsverfahren zeitaufwändig macht, die Produktivität verringert und die Korruption erhöht (LAHZ, 2003).

In einer Umfrage der Weltbank in 133 Ländern über die Staatsbürokratie und ihre Auswirkungen auf die Wirtschaft stellt sie klar, dass Zeit ein Faktor ist, der relevanter ist als die Kosten. Rita Ramalho, Direktorin der Weltbank: "Verlorene Zeit mit Bürokratie kostet Brasilien mehr als Steuern. Eine Vereinfachung des derzeitigen Steuersystems zur Erleichterung des Lebens des Steuerpflichtigen" (LIMA, 2017)

In einer Gewichtungsrangliste gilt Brasilien als eines der bürokratischsten, das 80 Tage für den Fortschritt des gesamten Prozesses in Betracht kommt.

deckt die historische Analyse des modernen Staates ab und argumentiert:

Es gibt Modernisierung, das kann man nicht leugnen, aber es ist eine Modernisierung, die als konservativ bezeichnet wird. Es ist diejenige, die Getélio Vargas getan hat, zu der auch das Militär getan hat, eine Modernisierung, die von oben kommt, wo der Staat extrem mächtig ist. Im aktuellen brasilianischen Fall geht es, wie auch weltweit, um diesen Staatsrücksetzer. Es ist ein Staat, der parallel zum soliden Aufbau einer Gesellschaft wieder aufgebaut werden muss. Im konkreten Fall Brasiliens muss der Staat wieder aufgebaut werden, aber es ist klar, dass wir dringend eine Gesellschaft aufbauen müssen. Nicht pessimistisch sein zu wollen, das wird nicht in einem magischen Pass geschehen, es ist nirgendwo auf der Welt geschehen oder geschehen; und es ist gut, 
dass es nicht passiert, denn wenn es passiert, ist es eine Sandburg, die jederzeit einstürzen wird. Aber es ist notwendig, diese Gesellschaft aufzubauen, obwohl es einige Zeit dauert, spielt es keine Roll[...]e. (PINHO, 2001p. 37)

Caio Marini (2003) beschäftigt sich in seinem Werk "Public management: contemporary debate" mit Nachhaltigkeit im Rahmen der staatlichen Modernisierung und erläutert:

[...] die aktuelle Debatte über Fr[...]agen der öffentlichen Verwaltung scheint einige der Grundprinzipien der aktuellen Agenda zu bekräftigen, wie z. B. Bürgerfokus, Transparenz, soziale Kontrolle, Bewusstsein für haushaltspolitische Verantwortung, Management für Ergebnisse, Ethik und Professionalisierung des Öffentlichen Bediensteten. Die große Herausforderung besteht darin, die Unumkehrbarkeit des Transformationsprozesses durch die Stärkung erfolgreicher Initiativen und die Neuausrichtung, die für die Einbeziehung neuer Themen notwendig ist, zu gewährleisten. (MARINI, 2003, p.82).

Laut Roesler (2017) funktionieren öffentliche Organisationen nicht, weil sie neoliberalen Ansprüchen nicht gerecht werden. Die Exekutive und der Nationalkongress haben zusammengearbeitet, um Reformen umzusetzen, die Finanzkapital begünstigen, ohne auf die Interessen und Bedürfnisse der Bevölkerung zu achten. Auch nach Ansicht des Magistrats sind die Reformen in mehreren Aspekten und Ergänzungen ein Rückschlag: "Der gesetzgeberische Horizont besteht darin, mehr soziale Rückschläge durch "Reformen" zu fördern, die die Würde des brasilianischen Bürgers untergraben." (ROESLER, 2017).

Die neoliberale Perspektive bestimmt, dass alles privatisiert werden kann, was mit mehreren öffentlichen Unternehmen und dem Erbe des Landes geschehen ist. Angesichts der Justiz berichtet der Richter heute von der Unentschlossenheit von Richtern, die eine "alternative Gerechtigkeit" zum Nachteil der Bundesverfassung 
anwenden, und die Staatsanwaltschaft handelt selektiv unter dem Vorwand der Korruptionsbekämpfung und der öffentliche Sicherheit (ROESLER, 2017)

Zusammenfassend wird darauf hingewiesen, dass die Verschlechterung des Images öffentlicher Organisationen kein aktuelles Problem ist, sondern angesichts der Politik der staatlichen Sozialleistungen fortbesteht und zunimmt. Diese Methode hat einen negativen Ruf für Regierungen und Regierungen realisiert. Dieses Szenario schadet ernsten und ehrlichen Männern, die in die Politik eintreten, um die Situation allmählich zu ändern. Hier ist die Desbildung der Bevölkerung, die ethisches und unethisches nicht unterscheiden kann.

\section{DAS THEATER UND DIE RECHTE}

Es gilt als eine künstlerische Spezies und Kunst als autarke Bewegung, die mit Vergnügen und Reflexion verbunden ist. Die Tragödie, seit Dem Anfang der Zeit, hat den Charakter des bürgerlichen Rituals als eine gesellschaftliche Versammlung, die das öffentliche Leben der Athener Pélis im 5. Jahrhundert bezeichnet.

Angesichts einer selektiven Analyse, die sich auf das Verständnis konzentriert, ist es möglich, einige Mittel der Korrelation, die rechtsrechtliche Erfahrung der Athener Gerichte, aufzuzeigen. Es ist notwendig, zwei Kategorien wiederherzustellen: die ausgearbeiteten Prozesse und die Themen in den Stücken eingefügt. In Bezug auf das Verfahren gibt es eine Analogie zwischen den Mitteln, die gefunden wurden, um die Durchführung von Tragödien zu gewährleisten, und den Verfahrenshandlungen der Athener Gerichte.

Laut Roland Barthes (1990) ist es wichtig, den zivilen Charakter des griechischen Theaters, vor allem der Tragödie, drastisch zu unterstreichen, da es die Stadt war, die ihr ihr Wesen zur Verfügung stellte. Die Beschreibung der Tragödie als Ergänzung der Dynamik von Pélis tritt nach Barthes (1990) auf.

Die Tragödien waren alle Gedenkfeiern, um den Gott Dionysos zu loben, und das Theater war für den Gottesdienst bestimmt. Um zu entscheiden, welche Gruppe die 
Tragödie in den Feierlichkeiten präsentieren würde, wurde ein Wettbewerb gemacht, wo der Archonde, Offizier der Pélis, war verantwortlich für die Vorbereitung der Schauspieler vor einer Gruppe von Juroren, die nach der Inszenierung entschieden, wer in der Ehrung handeln würde.

Die Choreographie war eine Schirmherrschaft, die von wohlhabenden Bürgern den Tragödien zugeschrieben wurde. Nach der Entscheidung des Wettbewerbs die Archonde, es benannt coregos. Ergänzungen (BARTHES, 1990, S.71) "Die finanziellen Belastungen waren hoch: Es lag an corego, den Proberaum zu mieten, die Ausrüstung zu bezahlen, den Darstellern Getränke zu liefern, die tägliche Vergütung der Künstler zu bezahlen".

Thééricon war eine Art Unterstützung, die von pélis geleistet wurde. Die Aufführungen waren ursprünglich kostenlos, aber die Zahl der Zuschauer stieg allmählich. In diesem Zusammenhang wurden zwei Obolos pro Tag der Barthes-Show (1990) in Rechnung gestellt. Alle diese Verfahren zur Auswahl, Förderung und Unterstützung des Stückes stellen die direkte Kontrolle des Plylis durch seine Organisationen und Vertreter dar.

Parallel dazu wird ein Verhalten der Athener Gerichte im 5. Jahrhundert überprüft, um die Forderungen zu lösen. Die Staatsbürgerschaft wurde nur auf freie erwachsene Männer ausgedehnt, die in der Stadt in Athener Familien geboren wurden, um festzustellen, dass Kinder, Frauen, Ausländer und Sklaven automatisch ausgeschlossen wurden. Es wird beobachtet, dass die Gerichte waren ausschließlich für Athener Bürger. Ebenso war die Regel die tragischen Stücke: Dichter und Schauspieler waren Athener Bürger.

Mehrere Elemente, die im Tragödienwettbewerb verwendet werden, finden entsprechende Verfahren vor Gericht. Helio, das wichtigste Athener Gericht, wie in den Ausschreibungen, bestand aus zehn Bürgern von Athen, mit einem jährlichen Mandat, in einem Verfahren, das von der Versammlung durchgeführt und für den Prozess von einem der Bogenschützen vorbereitet. (FINLEY,1991) 
Die Prozesse waren öffentlich und fanden in einem offenen Raum mit mündlichen Vorträgen beider Parteien statt, ohne Anwälte oder Staatsanwälte, und die Entscheidung lag bei den anwesenden Bürgern. (LOPES, 2000)

Als die politischen Institutionen der Athener begannen, wichtige Diskussionen und Fragen im Zusammenhang mit der Zukunft von Plyelis zu zentralisieren, begannen sich die Bürger sorgen sich um die Aristokratie, wohlhabende Bürger, die täglich an Einrichtungen teilnehmen konnten. unbeschadet ihrer Vergütung. Um die Beteiligung des Volkes zu verallgemeinern, ergriff Athen Maßnahmen wie Mistoforia, die ähnlich wie thé-ricon war: die tägliche Arbeit wurde an die Bürger gezahlt, um an den Sitzungen der öffentlichen Stellen teilzunehmen.

Von der Stadt kontrolliert und an öffentlichen Orten ausgeübt (SEGAL, 1994). Beide wurden von Richtern von Pélis vorbereitet, fehlte eine Auslosung, um die Jury zu komponieren, waren ausschließlich für die Bürger, zusätzlich zu der Zahlung für die Teilnahme der weniger begünstigten Klassen, wie von Vernant und Vidal-Naquet (1999) festgelegt.

Im Konzept von Barthes (1990) besteht der Zweck von Agon darin, Konflikte unparteiisch zu vermitteln. Durch tragödienweise stellt sich die Stadt selbst nach staatsbürgerlichen und bürgerfeindlichen Werten. (VERNANT E VIDAL-NAQUET, 1999)

In ähnlicher Weise diskutiert Tragödie Dilemmas rund um die Beratung, nach De Giorgi (2006). Solche Dilemmata werden der Öffentlichkeit präsentiert und auf der Bühne gelöst, während die Stadt jederzeit mit Beratungen konfrontiert ist. Die Tragödie wirft eine Frage nach seiner eigenen Identität auf, angesichts von Streitigkeiten, Sackgassen und Bindungen. Der autor fügt hinzu: "Die Mythen, die in der Tragödie präsentiert werden, spiegeln nicht bereits die traditionellen Werte einer fernen Ära wider, die idealisiert wurde. Im Gegenteil, sie werden zum Schlachtfeld der inneren Kämpfe der Stadt" (SEGAL, 1994, S. 195). 
Es sollte angemerkt werden, dass sich der Kern der Handlung um die Beratung dreht. Philosophen wie Goethe, Hegel und Lacan retteten den Streit des Zweifels. (ROSENFIELD, 2006). Die Untersuchung des Stücks umfasst mehrere Dilemmata wie: die Rivalität zwischen dem Gesetz der Götter und dem Gesetz der Menschen, unterstützt von Creonte; die Trennung zwischen Zivilisation und Natur; politischen Konflikt, um die Fortsetzung des Königreichs der Dynastie des Cadmeus zu verhindern. (ALMEIDA, 1997). Dieses Stück stellt einen Moment der Transformation der griechischen Religiosität selbst und die Reminiszenz mythologischer Narrative dar, die eine Regierung begünstigen, die auf menschlichen Kriterien und Logik basiert.

Sie benutzt mythische Narrative als Instrument, um wesentliche Fragen der Politik und des Rechts zu externalisieren. Die primären Einheiten der demokratischen Ply-lis, nach Rosenfield (2006).

Die Lehre des Gesetzes ist voll von Gewohnheiten und prosaceous, wie Hazing, Überwachung, die Simulation des Geschworenengerichts, unter anderem. Die Grenze zwischen Recht und Theater liegt in der Inszenierung. Während das Theater Rechtsfragen aufdeckt, die sich hauptsächlich auf die Menschenrechte konzentrieren, sind die Techniken der darstellenden Künste pädagogische Werkzeuge, um den Einfallsreichtum des zukünftigen Bachelor of Law zu unterstützen.

Im Bereich des Strafrechts ist es, wenn der Bachelor am meisten erkennt das Theater, weil es die Simulation von Urteilen wie die Gerichte der Jury, die Techniken der rechtlichen retoric, die das Oratorium umfasst, Intonation, Mimik und Bewegung im Raum, die theatralischen Inszenierungstechniken ähneln.

Das Drehbuch des Schauspielers Thiago Scalia in Zusammenarbeit mit Richter Henrique Macedo de Oliveira aus dem Jahr 2002 bezieht sich auf die diktatorische Ära, die von Getélio Vargas zwischen 1930 und 1945 kommandiert wurde. Das Stück zielte auf Menschenrechtsverletzungen.

Unter dem Vorsitz von Katia Bizzinoto im Jahr 2014 entstand die Kulturkommission, Schauspielerin und Theaterregisseurin mit dem Ziel, die Bemühungen um die 
Schaffung kultureller Rechte unter den brasilianischen Grundrechten durch die folgenden Richtlinien zu erweitern: Beitrag zur Bildung der kulturellen Bürgerschaft; Förderung von Aktivitäten, die sich auf kulturelle Themen konzentrieren; die Projekte von Gesetzen für kulturelle Zwecke zu kooperieren und zu beeinflussen und den Austausch zwischen Kunst und Recht zu ermöglichen.

In Rio de Janeiro hat die bundesstaatliche Bildungseinrichtung durch Anreize und Partnerschaften das Jurisdrama-Programm, das darauf abzielt, das Bewusstsein zu schärfen und individuelle und kollektive Rechte zu verteidigen, eine Aktion, die durch Theaterkurse und Repräsentationen Reflexion umfasst. in rechtlichen, sozialen und wirtschaftlichen Fragen, die den Anforderungen der Hochschulerweiterung auf der Grundlage von Lehre und Forschung gerecht werden.

\section{FAZIT}

Recht und Theater sind seit dem 5. Jahrhundert parallel, als Tribute an gottdionysosus, Gott der Freude, Fruchtbarkeit und Verschleierung, mit Gesängen und Inszenierungen gepriesen wurden. Solche Darstellungen überwanden den Charakter des Urteils, das in den Gerichten durch ihre Geschichten verwendet wird, so dass die Menschen ihre Meinung zu gemeinschaftlichen Angelegenheiten äußern konnten.

Es gab Wettbewerbe für die Auswahl von Gruppen, die sich in den Feierlichkeiten präsentieren würden, sowie der Rat, der eine bestimmte Forderung beurteilen würde. Unter anderem stellte das Theater den sozialen Charakter bestimmter Situationen dar, in denen Gesetze als fair oder unfair und unter Einhaltung der Menschenrechte analysiert wurden. Derzeit wird der Unterricht einiger Techniken der darstellenden Künste in einer Hochschule zunehmend eingeführt, so dass die Erfahrung des Studenten in seinem zukünftigen Beruf ermöglicht seine Leistung in Simulationen des Geschworenengerichts, zum Beispiel, zusätzlich zu anderen Aktionen, die, durch das Theater versuchen, rechtliche Fragen zu klären, Abdeckung der Analyse, gibt es den Film "The Breakdown of Time", ein Film, der die Realität vieler öffentlicher Ämter porträtiert. 
Die Krise, mit der der Staat konfrontiert ist, und die Glaubwürdigkeit derjenigen, die verschiedene öffentliche Organisationen leiten, erforderten einen internen Wiederaufbau, um die Eigentumsverhältnisse zu gewährleisten, und halfen nicht, die wirtschaftliche Koordinierung auf dem Markt zu unterstützen und Ungleichheiten zu verringern. sozial.

Von hier aus wurden öffentliche Einrichtungen analysiert, als Schwerpunkt auf Stiftungen und versuchten, den 1995 aufgestellten Staatsreformplan zu untersuchen. Das brasilianische öffentliche Management hat in den letzten zehn Jahren große Veränderungen durchgemacht, um eine größere Effizienz der öffentlichen Dienstleistungen zu erreichen. Der Reformplan-Ansatz zielt darauf ab, die Staatsmaschineschneller, flexibler und bereit zu machen, soziale Bedürfnisse mit einer Management-Herausforderung zu erfüllen. Die Hauptaufgabe dieses Plans besteht darin, Die Grundprinzipien so zu formulieren, dass die Ziele der Ausweitung der staatlichen Governance das staatliche Handeln auf die Funktionen beschränken, die ihr eigener Übergang von der Union zu den lokalen Aktivitäten der Kommunen sind, und teilweiseÜbertragung von der Union auf die Staaten können regionale Aktivitäten vollständig eingerichtet werden.

Die Reform des öffentlichen Sektors auf der Suche nach Qualität in der Erbringung von Dienstleistungen schlug die Dezentralisierung einiger öffentlicher Organisationen und um diese Dekonzentration umzusetzen, waren Änderungen bei der Durchführung der Von den Servern durchgeführten Tätigkeiten erforderlich. Öffentlich. Zu den Leitlinien, die die von der Reform festgelegten Ziele leiten, gehören die Neuorganisation öffentlicher Einrichtungen und eine Änderung der Standards. Dieser Vorschlag zielt darauf ab, Versäumnisse und Maßnahmen des Staates neu zu definieren und signifikante Änderungen in der Verwaltung öffentlicher Einrichtungen zu erreichen, so Silva und Fadul (2010).

Die Krise, mit der der Staat konfrontiert ist, und die Glaubwürdigkeit derjenigen, die verschiedene öffentliche Organisationen leiten, erforderten einen internen Wiederaufbau, um die Eigentumsverhältnisse zu gewährleisten, und halfen nicht, die 
wirtschaftliche Koordinierung auf dem Markt zu unterstützen und Ungleichheiten zu verringern. sozial. Die Globalisierung hat es relevant gemacht, öffentliche Dienstleistungen neu zu definieren. Nach innen hatte die globale Einbeziehung von Märkten und Produktivmodi die Staaten als eines ihrer grundlegenden Ziele, ihre Volkswirtschaften vom internationalen Wettbewerb zu schützen. Nach dem Globalisierungsprozess konnte der Staat diese Haltung nicht beibehalten, sondern ein starkes internes Wirtschaftssystem einführen, das ihn international wettbewerbsfähig macht.

Erste Verwaltungsreform auf der Grundlage beruflicher Verdienste. Die bürokratische öffentliche Verwaltung wurde eingerichtet, um die Verwaltung der Patritrionen zu ersetzen, die absolute Monarchien kennzeichnete, die die Grenze nicht unterschieden. In diesem Modell der Verwaltung war der Staat eigentum des Königs, ebenso wie Vetternwirtschaft und Korruption üblich waren.

Diese Art der Verwaltung ist antagonistisch, es ist zwingend notwendig, dass der Kapitalismus den Staat trennt und der Markt und die Demokratie nur existieren, wenn sich die Zivilgesellschaft vom Staat abstreitt und sie gleichzeitig kontrolliert. Es war notwendig, eine unparteiische Verwaltungsform zu entwickeln, die in diesem Zusammenhang auf ein modernes bürokratisches Management entsteht.

Nach und nach wurden die Grundlagen der neuen öffentlichen Verwaltung geschaffen, indem die Grundlage für die politische Dezentralisierung geschaffen wurde, wobei Ressourcen und Vorrechte auf regionale und lokale politische Bereiche übertragen wurden; administrative Dezentralisierung durch die Zuweisung von Befugnissen an öffentliche Manager, die in autonome Manager umgewandelt wurden; Entitäten mit kleineren Hierarchien; die Annahme eines begrenzten Vertrauens; und Auswertung durch Ergebnisse.

Die durch das Dekret 200/67 eingeführte Reform war ein Versuch, bürokratische Starrheit zu überwinden, und kann als erste Management-Management in Brasilien betrachtet werden. Der Kern zielte auf die Dezentralisierung angesichts der Autonomie 
des indirekten Managements ab. Die Organisation, das Budget, die Dekonzentration und die Auswertung der Ergebnisse wurden als Grundlage für die Rationalität der Verwaltung festgelegt.

In den dezentralen Basen wurden celetistas-Mitarbeiter eingesetzt, die dem privaten Vertragssystem untergeordnet waren. Die Periode begünstigte den Ausbau der öffentlichen Unternehmen. Die Flexibilität seiner Verwaltung wurde angestrebt, um eine größere Wirksamkeit der wirtschaftlichen Praktiken des Staates zu erreichen und den politischen Pakt zwischen staatlicher, ziviler und militärischer Technologiebürokratie und der Wirtschaft zu stärken.

Dieses Dekret hatte zwei unerwartete Ergebnisse. Durch die Zustimmung zur Einstellung von Arbeitnehmern ohne öffentliche Ausschreibung erleichterte sie die Aufrechterhaltung von Patritrionen-Praktiken und hielt keine Auswahlverfahren oder Karrierepläne ab. Das strategische Zentrum des Staates war abgenutzt, begünstigte die Einstellung der hohen Führungsebenen durch staatliche Unternehmen. Der reformpolitische Versuch des Dekrets 200 scheiterte. In den 1970er Jahren verschärft die Krise des Militärregimes die Landschaft der öffentlichen Verwaltung weiter, während die Staatsbürokratie mit dem autoritären Modell im Prozess der Degeneration anerkannt wird.

Der demokratische Übergang im Jahr 1985 war kein günstiger Horizont für reformen der Staatsmaschine. Im Gegenteil, sie bedeutete eine Rückkehr zu früheren bürokratischen Ideologien im administrativen Bereich, während sie im politischen Bereich eine Rückkehr zum Populismus darstellte. Zu Beginn des demokratischen Regimes wurden die Haushaltskrise und die wirtschaftliche Intervention außer Acht gelassen, das Importaustauschsystem beibehalten und die Gehälter und öffentlichen Ausgaben stiegen, Faktoren, die zum Scheitern des Kreuzfahrerplans führten. In der Folge gab es einen Versuch einer Haushaltsanpassung, der durch die mangelnde Unterstützung der brasilianischen Gesellschaft vereitelt wurde. Gleichzeitig etablierte die konservative politische Koalition im Kongress eine populistische und patrimoniaistische Politik. 
Die Verfassung von 1988 war eine Folge dieser widersprüchlichen Bewegungen. Die Magna Carta ist eine Antwort auf den Populismus, der auf Demokratie zurückgeht, und die Grundlagen archaischer und bürokratischer öffentlicher Verwaltung mit zentralisierter, hierarchischer und unflexibler öffentlicher Verwaltung realisiert. Die Gesellschaft und die Wähler zeigten einen Mangel an Innovationsfähigkeit, da sie die dezentralisierten und flexiblen Modelle, die im Dekret 200 als Ergebnis einer effizienten staatlichen Verwaltung verankert sind, nicht bemerkten.

Der bürokratische Rückschlag, den die Verfassung von 1988 mit sich brachte, war eine Haltung des Widerstands gegen den Klientelismus, die das Land damals heimsuchte, aber auch die korporatistischen und patrimoniaistischen Privilegien ratifizierte, die dem bürokratischen Ethos entgegenstanden. Diese Elemente begünstigten die Verschlechterung der öffentlichen Verwaltung in Brasilien, obwohl die Kompetenz und Ehrlichkeit der brasilianischen öffentlichen Verwalter berüchtigt sind.

Die fiskalische und interventionistische Instabilität entstand 1987. Nur mit Hyperinflation während José Sarneys Amtszeit Gesellschaft erkannte die Schwere der Krise, in der Berufung auf Maßnahmen, in den Verhandlungen über den Ausgleich der Wirtschaft, die Aufmerksamkeit von Fernando Collor Regierung gewonnen, in dieser unruhigen Zeit tritt auf kommerzielle Offenheit, mit Privatisierung. Haushaltsanpassungen erhalten permanente Leitlinien, und das Land erreicht einen breiten Schuldenerlass für das Inland.

Im Rahmen der öffentlichen Verwaltung haben die Reformen einen anderen Verlauf genommen. Dieses Scheitern war hauptsächlich auf den ungeschickten Versuch zurückzuführen, die Staatsmaschinerie zu reduzieren, Beamte zu entlasten und Organe zu schließen, ohne sich erneut mit der Rechtmäßigkeit der Maßnahmen zu beschäftigen. Es gab eine Gehaltskürzung der Server und desorganisiert die bestehende bürokratische Struktur, entehren die Beamten, durch Korporatismus anerkannt (Schutz der Interessen in einer Gruppe, als ob die Interessen der Nation vertreten). 
Die Itamar-Regierung, (nach der Amtsenthebung), mit der schwierigen Mission, angesichts eines Szenarios, der Rezession, diskreditieren, Mangel an Vertrauen in die Regierung, (intern und extern), leiden unter den Folgen, der ineffektiven Verwaltung. Im ersten Jahr hatte es die technische Kapazität des Wirtschaftsteams des Finanzministeriums zu dieser Zeit, zusammen mit dem Wunsch, öffentliche Konten und Markttrusts und Menschen wieder ins Gleichgewicht zu bringen. So beginnen Maßnahmen, die auf wissenschaftlichen Studien basieren, den Ursprung der Karriere von öffentlichen Managern, die sich auf Public Policies und Government Management spezialisiert haben.

Der öffentliche Dienst ist ineffektiver, kostspieliger und autonomer geworden. Die Spaltung wurde nicht nur durch das patrimonialistenmodell vorbereitet, sondern auch durch die Einführung eines einheitlichen Rechtssystems, mit der Entlastung der Zeletisten und der verfassungsmäßigen Behauptung eines starren Stabilitätsmodells, bei dem die Bewertung der Serverleistung Diese Stabilität des öffentlichen Funktionalismus ist ein inhärenter Aspekt des bürokratischen Managements und wurde geschaffen, um die Diener und den Staat selbst zu schützen. In Brasilien, während der Kaiserzeit, als eine Regierung abgesetzt wurde, verloren viele vertrauenswürdige Beamte, zusätzlich zu anderen gewöhnlichen Menschen ihre Positionen.

Stabilität hat jedoch Kosten und verhindert die Anpassung des Serverframeworks an die tatsächlichen Anforderungen des Dienstes. Andererseits war es unmöglich, ein wirksames öffentliches Managementsystem einzuführen, das auf einem Modell von Anreizen und Strafen beruht.

Es wurden drei relevante Faktoren angesprochen, die die Verschlechterung des Images öffentlicher Institutionen beeinflussen, wie Korruption, brasilianische Kultur und Ineffizienz des Managements.

Die brasilianische Ausbildung war schon immer prekär, da sie auf das Diplom und nicht auf das Wissen abzielte. Die Kultur der Indianer und Schwarzen war eingeengt und 
diese durften nicht lernen, diese Divergenz zwischen fortgeschrittener politischer Abstraktion und der Verzögerung der Umwelt zeigte den Punkt der kulturellen Desorientierung, die Völkern mit kolonialen Vorfahren innewohnt.

In der Mitte des 21. Jahrhunderts hat Brasilien nach IBGE-Daten eine Analphabetenrate von $7 \%$ bei Menschen ab 15 Jahren.( Ein Index, der noch weit von dem der Industrieländer und einigen in der Entwicklung entfernt ist. Das Panorama der Desbildung der brasilianischen Bevölkerung verhindert eine größere Einmischung in die Überlegungen des Systems, aber die Gesellschaft erwacht allmählich, vielleicht durch das Aufkommen des Internets oder durch Müdigkeit angesichts des Bildungsungleichgewichts.

Korruption ist heute eines der am meisten kommentierten Themen und wird von Wissenschaftlern als moralische und ethische Frage betrachtet. Bezerra (1995) konzeptioniert Korruption als "Phänomen struktureller Natur, das in unserer sozialen Bildung, unseren Gewohnheiten und Bräuchen verwurzelt ist". Um das Problem zu lösen, sollten strengere und ethischere moralische Standards festgelegt werden, so der Autor. Aus soziologischer Sicht ist es zwingend zu prüfen, dass debatten über soziale Bedingungen, die zum Lebensunterhalt und zur Erneuerung der Korruption im Land beitragen, aufgegeben werden, indem moralische Prinzipien als Grundlage für dieses Phänomen herangeführt werden. (BEZERRA, 1995). Um dieses Panel zu ändern, ist das Handeln der gesamten Gesellschaft in der Konfrontation gegen die Korruption sehr relevant. Die ständigen sozialen Anstrengungen und die Verurteilung von Korrupten können große Veränderungen zur Verringerung oder sogar Ausrottung der Korruption im Land mit sich bringen (STUKART, 1988).

In Bezug auf die Ineffizienz von Führungskräften ist es erwähnenswert, dass Managementmethoden wichtige Werte wie Qualitätsproduktivität und Verantwortung festlegen sollten. Wie bereits erwähnt, hat die bürokratische Krise der brasilianischen öffentlichen Verwaltung ihren Ursprung im Militärregime, angesichts des Erbumtums, der die Rekrutierung von Managern durch staatliche Unternehmen ermöglichte. Es gibt Unterschiede zwischen der Beschäftigung durch die öffentliche Maschine des 
Hybridverwaltungssystems des Staates, d. h. von Einrichtungen, die Dienstleistungen mit Unterstützung der Steuererhebung, aber auch mit privatem Sponsoring erbringen. Hinzu kommt die Bürokratie, an der öffentliche Organisationen beteiligt sind. Der Mangel an Investitionen in Technologie beeinträchtigt die höhere Geschwindigkeit in öffentlichen Dienstleistungen, da Online-Verfahren $74 \%$ weniger kosten als persönliche Verfahren, um sie abzuschließen und niedrigere Kosten zu verursachen.

Eine kurze Analogie zwischen Theater, seinen Charakteren und öffentlichen Institutionen. Öffentliche Organisationen sind Teile ohne definiertes Skript, da die festgelegten Richtlinien ihre Vorgänge ändern. Auf der Suche nach der Öffentlichkeit versuchen die Akteure, ihr Bestes zu geben, werden aber durch Infrastrukturprobleme verhindert, da die Zunahme der Geschwindigkeit des Funktionalismus mit Bürokratie konfrontiert ist, die die Nutzer behindert. Auf der anderen Seite sieht sich der Regisseur die Hände gebunden, da er sich den Sponsoren beugen sollte. Ineffizienz in der Unternehmensführung ist auch eine Folge der vom Staat eingeleiteten Politik und aller Verfahren, mit denen der Leiter einer öffentlichen Einrichtung konfrontiert ist, um bessere Arbeitsbedingungen oder Einnahmen zu erreichen.

Das Wichtigste ist, dass dieses Stück mit einem definierten Drehbuch, guten und effizienten Schauspielern, mit ständigem Sponsoring und zufriedenen Zuschauern, unabhängig von der Zeit, ausgestellt werden soll.

\section{REFERENZEN}

ADMIN. Fundações Públicas e Privadas: Equiparação: Inconstitucionalidade. 2008. Disponível em:< http://www.altosestudos.com.br/?p=41230>. Acesso em: 06 dez 2019.

ALMEIDA, G; VIEIRA, T. Três tragédias gregas: Antígona, Prometeu prisioneiro e Ájax. São Paulo: Perspectiva. 1997

ANDRADE, Regis de Castro. Estrutura e organização do Poder Executivo frente à opção pelo sistema de governo: Estrutura da Administração Pública e gestão de

Disponível em: https://www.nucleodoconhecimento.com.br/business-administration-de/das- 
recursos humanos em quatro países parlamentaristas da Europa: França, GrãBretanha, Espanha e Itália. São Paulo: Cedec, 1993.

ANDRADE, Regis de Castro; JACCOUD, L. Estrutura e organização do poder Executivo. Brasília: ENAP/SAF, vol. I. 1993

ARAGÃO, Cecilia Vescovi de. Burocracia, eficiência e modelos de gestão pública: um ensaio. $1997 . \quad$ Disponível em:< http://www.bresserpereira.org.br/Documents/MARE/Terceiros-Papers/97Arag\%C3\%A3o,CVescovide48(3).pdf>. Acesso em: 06 dez 2019.

BARTHES, R. O teatro grego. In. O óbvio e o obtuso - ensaios críticos III. Trad. Léa Novaes. Rio de Janeiro: Nova Fronteira. 1990

BEZERRA, Marcos Otávio. Corrupção: um estudo sobre poder público e relações pessoais no Brasil. Rio de Janeiro: Relume Dumará, 1995.

BID (Banco Interamericano de Desenvolvimento) e OCDE (Organização para a Cooperação e Desenvolvimento Econômico). 2016. Government at a Glance 2017. París: OCDE. Disponível em: http://dx.doi.org/10.1787/gov_glance-2017-en.. Acesso em: 06 dez 2019

BOBBIO, Norberto. A era dos direitos. Rio de Janeiro: Campus, 1992.

BRASIL. Constituição da República Federativa do Brasil, 1988. Disponível em: $<$ https://www2.senado.leg.br/bdsf/bitstream/handle/id/518231/CF88_Livro_EC91_20 16.pdf >. Acesso em: 06 dez 2019.

BRASIL. Decreto lei 200/67. Disponível em: <https://presrepublica.jusbrasil.com.br/legislacao/104099/decreto-lei-200-67>. Acesso em: 06 dez 2019.

BRASIL. Plano Diretor da Reforma do Aparelho do Estado. Brasília: 1995. Disponível $\quad$ em:< $\quad$ http://www.biblioteca.presidencia.gov.br/publicacoes- 
oficiais/catalogo/fhc/plano-diretor-da-reforma-do-aparelho-do-estado-1995.pdf Acesso em: 06 dez 2019.

$\begin{array}{lccc}\text { BRASIL. Burocracia e Politicas Públicas no Brasil: interseções analíticas. } \\ \text { Brasília, } & 2018 . & \text { Disponível }\end{array}$ <http://repositorio.enap.gov.br/bitstream/1/3247/1/livro_Burocracia\%20e\%20pol\%C3 \%ADticas\%20p\%C3\%BAblicas\%20no\%20Brasil\%20\%20interse\%C3\%A7\%C3\%B5es\%20anal\%C3\%ADticas.pdf>. Acesso em: $06 \mathrm{dez}$ 2019.

BRASIL. Jurisdrama. Disponível em:<http://www.jurisdrama.ufrj.br/>. Acesso em: 06 dez 2019.

CALEGARI, Luiza. Percepção de corrupção no Brasil é a pior em cinco anos. Revista Exame. Disponível em: < https://exame.abril.com.br/brasil/percepcao-decorrupcao-no-brasil-e-a-pior-em-cinco-anos/> Acesso em 06 dez. 2018.

CARVALHO FILHO, José dos Santos. Manual de Direito Administrativo. Rio de Janeiro: Lumen Juris $21^{\circ} \mathrm{ed} .2009$

CARVALHO, Virginia Donizete de; OLIVEIRA, Thais Aluxe de; SILVA, Daniele Cristhiane da. Valores organizacionais em instituições públicas brasileiras: percepções dos servidores em diferentes posições hierárquicas e tipos de entidade da administração indireta. 2013. Disponível em:< http://www.scielo.br/pdf/ram/v14n5/04.pdf >. Acesso em: 06 dez 2019.

DE GIORGI, R. Direito, tempo e memória. Trad. Guilherme Leite Gonçalves. São Paulo: Quartier Latin, 2006.

DINIZ, Gustavo Saad. Direito das Fundações Privadas - Teoria Geral e Exercício das Atividades Econômicas. 2. ed. Porto Alegre: Síntese, 2003. 
FERLIE, Ewan; ASBURNER, Lynn; FITZGERALD, Louise; PETTINGREW, Andrew. A nova administração pública em ação. Trad. Sara Rejane de Freitas Oliveira. Brasília: ENAP, 1999.

FERRARI, Hamilton. Correio Braziliense. Burocracia: setor público leva 5,5 horas para realizar único procedimento 2018. Disponível em: $<$ https://www.correiobraziliense.com.br/app/noticia/economia/2018/06/11/internas_ec onomia,687669/burocracia-setor-publico-leva-5-5-horas-para-realizar-unicoprocedime.shtml>. Acesso em: $06 \mathrm{dez} 2019$.

FINLEY, M.I. Aspectos da Antiguidade. Trad. Marcelo Cipolla. São Paulo: Martins Fontes, 1991.

JUSTEN FILHO, Marçal. Curso de Direito Administrativo. São Paulo: Saraiva, 2008.

LAHÓZ, André. Liberem o Crescimento. Revista EXAME, São Paulo, № 23, ano 37, ed. 805, 2003.

LIMA, Luís. Tempo perdido com burocracia custa mais ao Brasil que impostos, diz diretora do Banco Mundial. 2017. Disponível em: <https://epoca.globo.com/economia/noticia/2017/09/custo-do-tempo-pesa-mais-queo-financeiro-diz-diretora-do-banco-mundial-sobre-burocracia-brasileira.html>. Acesso em: 06 dez 2019.

LOPES, J.R. Lima 0 direito na história: lições introdutórias. São Paulo: Max Limonad. 2000

MARINI, Caio. Gestão Pública: O Debate Contemporâneo. Cadernos da Fundação Luís Eduardo Magalhães. № 07. Salvador: Fundação Luís Eduardo Magalhães, 2003. 
MAZILLI, Johnny. Revista Planeta. O que é que a Coreia tem? 2011. Disponível em: $<$ https://www.revistaplaneta.com.br/o-que-e-que-coreia-tem/>. Acesso em: $06 \mathrm{dez}$ 2019.

MENDONÇA, Saulo Bichara. Quimera da eficiência no serviço público desestruturado. $2017 . \quad$ Disponível em:< https://professorsaulo.jusbrasil.com.br/artigos/457823003/quimera-da-eficiencia-noservico-publico-desestruturado?ref=topic_feed >. Acesso em: 06 dez 2019.

MONTEIRO, Carolina. Correio de Uberlândia. Teatro ajuda futuros juristas na formação e cumpre papel social. 2018. Disponível em: $<$ http://www.correiodeuberlandia.com.br/entretenimento/teatro-ajuda-futuros-juristasna-formacao-e-cumpre-papel-social/>. Acesso em: 06 dez 2019.

MOTTA, P. R. A modernização da administração pública brasileira nos últimos 40 anos. Revista de Administração Pública, v. 41, n. esp., p. 87-96, 2007.

MOTTA, F. C. P.; VASCONCELOS, I. F. G. Teoria geral da administração. 3. ed. São Paulo: Thomson Learning, 2006.

OLIVEIRA. Nielmar de. Agência Brasil. IBGE: taxa de analfabetismo cai 0,2 ponto percentual em 2017. 2018. Disponível em: <http://agenciabrasil.ebc.com.br/economia/noticia/2018-05/ibge-taxa-deanalfabetismo-no-pais-cai-02-ponto-percentual-em-2017>. Acesso em: 06 dez 2019.

OSBORNE, David; GAEBLER, Ted. Reinventando o Governo. Tradução Sérgio Bath e Ewandro Magalhães Júnior. Brasília: MH Comunicação, 1994.

PINHO, Antônio. Evolução do Estado Moderno. In: MACHADO, Geraldo et al. Gestão Pública: Desafios e Perspectivas. Cadernos da Fundação Luís Eduardo Magalhães. n 01. Salvador: Fundação Luís Eduardo Magalhães, 2001.

PEREIRA, Luiz Carlos Bresser. Da administração Pública burocrática à gerencial. Revista do Serviço Público: ENAP, ano 47, v. 120, 1996. 
PEREIRA, Luiz Carlos; SPINK, P.K (Orgs.) Reforma do Estado e Administração

Pública Gerencial. Ed. Rio de Janeiro: Fundação Getúlio Vargas, 1998.

PEREIRA, Luiz Carlos; SPINK, P. Reforma do Estado e Administração Pública Gerencial. 3. Ed. Rio de Janeiro: Fundação Getúlio Vargas, 1999.

PORTAL EDUCAÇÃO. A História do Teatro no Brasil e no mundo. Disponível em: $<$ https://www.portaleducacao.com.br/conteudo/artigos/direito/historia-do-teatro-nobrasil-e-no-mundo/50069>. Acesso em: 06 dez 2019.

RAYOL, Paulo André Araújo. Fundações públicas e privadas. 2017. Disponível em: $<$ https://jus.com.br/artigos/59258/fundacoes-publicas-e-privadas >. Acesso em: 06 dez 2019.

REALE, Miguel. Filosofia do Direito. 20. Ed. São Paulo: Saraiva. 2009

RIZZO, Sérgio. Crítica: A Repartição do tempo. 2018. Disponível em: $<$ https://oglobo.globo.com/rioshow/critica-reparticao-do-tempo22349905\#ixzz5PfxLOq4X>. Acesso em: 06 dez 2019.

ROESLER, Átila da Rold Justificando. As instituições Públicas não estão funcionando. Disponível em: < http://justificando.cartacapital.com.br/2017/12/13/asinstituicoes-publicas-nao-estao-funcionando/ > Acesso em: 06 dez 2019.

ROSENFIELD, K.H. Introdução, Comentários e notas. In: Sófocles. Antígona. Trad. Lawrence Flores Pereira. Rio de Janeiro: Topbooks. 2006

SCHAUN, Raimundo. Comunicação, Poder e Democracia. São Paulo: IPCJE, 1986.

SEGAL, C. O ouvinte e o espectador. In: Vernant, J-P. (org.). O homem grego. Trad. Maria Jorge Vilar de Figueiredo. Lisboa: Editorial Presença. 1994 
SENADO. Jus Brasil. Ana Amélia defende fortalecimento de instituições públicas. 2013. Disponível em:< https://senado.jusbrasil.com.br/noticias/100486565/anaamelia-defende-fortalecimento-de-instituicoes-publicas>. Acesso em: 06 dez 2019.

SILVA, Camila Garcia da. Revista Liberdades. O caso dos Irmãos Naves. Disponível em:

$<$ http://www.revistaliberdades.org.br/site/outrasEdicoes/outrasEdicoesExibir.php?rco n_id=58>. Acesso em: 06 dez 2019.

SILVA, L. P.; FADUL. A produção científica sobre cultura organizacional em organizações públicas no período de 1997 a 2007: um convite à reflexão. Revista de Administração Contemporânea, v. 14, n. 4, art. 5, p. 651-669, 2010.

SODRÉ, Nelson W. Síntese de história da cultura brasileira. $18^{\circ}$ ed. Rio de Janeiro: Bertrand Brasil, 1996.

STUKART, Herbert L. Ética e Corrupção nas Empresas com Enfoque sobre Compras. Salvador: ABAM - Associação Brasileira de Administração de Material. Caderno de Administração de Materiais. № 05, 1988.

SUPREMO TRIBUNAL FEDERAL. ADI 191-4. Relatora Ministra Carmen Lúcia. Disponível< file://C:/Users/CLIENTE/Downloads/STF\%20\%20ADI\%20n\%C2\%BA\%20191\%20RS\%20(2).pdf > Acesso em.: 06 dez 2019.

TEIXEIRA, Glória. Direito e Estudos Teatrais. In: Teatro do Mundo. Universidade do Porto. 2018. Disponível em: <http://ler.letras.up.pt/uploads/ficheiros/10182.pdf> Acesso em: 06 dez 2019.

VERNANT, J-P e VIDAL-NAQUET, P. Mito e tragédia na Grécia antiga I e II. Vv. trad. São Paulo: Perspectiva. 1999

Eingereicht: November 2019.

Genehmigt: Januar 2020. 